\title{
Sobre a formação de diminutivo do português brasileiro
}

\author{
Seung-Hwa Lee \\ Universidade Federal de Minas Gerais
}

\section{Abstract}

Diminutive formations are very productive in Brazilian Portuguese (BP). There are two ways to derive diminutive forms out of nominal words (i.e. nouns and adjectives/adverbs): -inbo(a) suffixation e -zinho(a) suffixation. The latter is attached at the stem with the thematic vowel $(-a,-o,-e$, which represent the morphological class in nominal words, like gender and number) and the former is attached at the stem without the thematic vowel.

There are many divergences in the literature to explain the diminutive formations which are treated: i) as derivational suffixes; ii) as phonological words; iii) -inho suffixation as derivation, -zinho suffixation as (phonological) compounding, etc.

In this paper, I will argue that diminutive formation in Brazilian Portuguese (BP) is treated differently from the derivation/ compound formation and the inflection in the process of word formation - that is, diminutive formation has independent status. 


\section{INTRODUÇÃO}

formação de diminutivo é muito produtiva no Português
Brasileiro (doravante, $\mathrm{PB}$ ), que exibe duas maneiras de
derivar as formas de diminutivo das palavras não-verbais (i.e. nomes e adjetivos/advérbios): a sufixação -inho(a) e a sufixação -zinho(a). O primeiro sufixo é acrescido aos radicais com vogais temáticas $(-a,-o,-e$, que representam, nas palavras não-verbais, classes morfológicas, como número e gênero), enquanto o segundo sufixo é acrescido aos radicais sem vogais temáticas. Na literatura, há divergências quanto à explicação para os sufixos de diminutivo, que são tratados como: i) sufixos derivacionais; ii) palavras fonológicas; iii) derivação por sufixação -inho(a) e composição fonológica por sufixação -zinho(a); etc. (cf. Câmara, 1970; Leite, 1974; Moreno, 1977; Brakel, 1981; Lee, 1992; Menuzzi, 1993).

Este trabalho apresenta uma nova proposta para a análise dos diminutivos no PB e argumenta-se que a formação de diminutivo no PB deve ser tratada diferentemente da derivação/composição e da flexão nos processos de formação de palavras, adaptando o modelo da Fonologia Lexical Prosódica (FLP, Inkelas, 1989, 1993).

\section{ALGUMAS PROPRIEDADES DO DIMINUTIVO}

Os morfemas do diminutivo, -inho(a) e -zinho(a), podem ser afixados às formas não-verbais, como mostra (1):

(1) a. casa $\rightarrow$ casinha, bonita $\rightarrow$ bonitinha

b. menino $\rightarrow$ menininho, perto $\rightarrow$ pertinho

c. café $\rightarrow$ cafezinho

d. flor $\rightarrow$ florzinha 
De modo geral, na formação de diminutivo, o morfema -inho é afixado a uma forma não-verbal contendo marcador de palavra, como ocorre em (1a, b), e o morfema -zinho é afixado a uma forma não-verbal sem o marcador de palavra, como se verifica em (1c, d). Nas palavras proparoxítonas e nas palavras que terminam em sílaba pesada, o diminutivo é formado através da afixação do morfema -zinho, como mostra (2): ${ }^{1}$

(2) a. lâmpada $\rightarrow$ lampadazinha número $\rightarrow$ numerozinho

b. judeu $\rightarrow$ judeuzinho troféu $\rightarrow$ trofeuzinho irmão $\rightarrow$ irmãozinho

c. mar $\rightarrow$ marzinho sol $\rightarrow$ solzinho

Além disso, os diminutivos apresentam algumas caraterísticas, que podem ser observadas a seguir:

i) A afixação de -inho(a), -zinho(a) não muda os traços do radical derivacional, tais como os traços de categoria lexical e gênero, o que é atestado pelos exemplos de (3):

(3) a. a $\operatorname{mesa}_{\mathrm{N}} \rightarrow$ a mesinha ${ }_{\mathrm{N}}$, $\mathrm{O}_{\operatorname{mar}_{\mathrm{N}}} \rightarrow \mathrm{o} \mathrm{marzinho}_{\mathrm{N}}$

b. velho $_{\mathrm{A}} \rightarrow$ velhinho $_{\mathrm{A}}$ fácil $_{\mathrm{A}} \rightarrow$ facilzinho $_{\mathrm{A}}$

c. o dente $\rightarrow$ o dentinho a sorte $\rightarrow$ a sortinha

d. a casa $\rightarrow$ o caseiro o livro $\rightarrow$ a livraria 
Os exemplos de (3 a, b, c) mostram que a categoria lexical e o gênero da palavra resultante do acréscimo dos sufixos são determinados pelos traços do radical, diferentemente do que ocorre no processo derivacional, no qual, a categoria lexical e o gênero são determinados pelo constituinte à direita, como em (3d). Isso significa que os sufixos -inho e -zinho, diferentemente dos sufixos derivacionais, não contêm os traços de categoria e de gênero.

ii) No processo derivacional, a qualidade das vogais médias, /E, O/, na posição tônica, muda para [e, o] na posição átona, durante a derivação, como ilustra (4):

a. b[É]lo $\rightarrow$ b[e]leza

caf[É] $\rightarrow$ caf[e]teira

b. $\mathrm{p}[\hat{O}] \rightarrow$ p[o]eira

$\mathrm{d}\left[\right.$ Ó]lar $\rightarrow \mathrm{d}[\mathrm{o}]$ larização ${ }^{2}$

Este processo é conhecido na literatura como neutralização vocálica (Wetzels, 1989, 1991, 1997). Essa neutralização, no entanto, não ocorre na formação produtiva do diminutivo, embora se aplique na posição átona, como mostra (5):

(5) a. b[Ó]la $\rightarrow$ b[O]linha

b. v[É]la $\rightarrow$ v[E]linha

c. caflÉ] $\rightarrow$ caf[E]zinho

d. b[É]la $\rightarrow$ b[E]linha

Além disso, outras formações produtivas encontradas no PB também não apresentam essa neutralização vocálica, conforme ilustram os exemplos de (6):

(6) a. b[E]lamente

b. b[E]líssima

c. d[O]larzinho 
iii) Há somente um acento lexical em palavras com os sufixos -inho(a), -issimo(a), ao passo que há mais de um acento lexical ${ }^{3}$ em palavras com os sufixos -zinho(a), -mente (cf. Brakel, 1981):

(7) a. sapatínho

belíssimo

b. rigoròsaménte

càfezínho

iv) A palavra com o sufixo -zinho permite o morfema plural entre o radical derivacional e o sufixo, ${ }^{4}$ enquanto a palavra com o sufixo -inho não permite, conforme exemplifica (8), abaixo:

(8) a. hotelzinho $\rightarrow$ hoteizinhos marzinho $\rightarrow$ marezinhos

b. casinhas, *casasinhas

\section{FORMAÇÃO PRODUTIVA E DOMÍNIO PROSÓDICO}

Apesar das semelhanças entre -inho e -zinho, em análises anteriores sobre a formação do diminutivo no PB (cf. Câmara, 1970; Leite, 1974; Moreno, 1977; Brakel, 1981; Lee, 1992), -inho é considerado como um sufixo (ou seja, um elemento que faz parte de um processo derivacional), ao passo que -zinho é considerado como um elemento que faz parte de um composto.

Nesta seção, a partir das análises anteriores, discute-se o domínio prosódico da formação produtiva do diminutivo do PB, à luz do modelo da Fonologia Lexical Prosódica (FLP, Inkelas, 1989, 1993), no qual a morfologia é distinta e separada da fonologia, mas as regras fonológicas aplicam-se nos objetos criados pela morfologia, a não ser que haja falta de isomorfia entre as estruturas morfológicas e as estruturas fonológicas. Essa falta da isomorfia pode ser explicada pela Hipótese de Referência Indireta, ${ }^{5}$ introduzindo a noção de domínio prosódico no léxico. Os domínios prosódicos são formados 
pela Formação de Constituinte Prosódico (PCF), derivado pela Formação de Constituinte Morfológico (MCF), que mantém as idéias da Hipótese de Nível Ordenado (Siegel, 1974). Em outras palavras, as regras fonológicas aplicam-se nos domínios prosódicos, mas esses domínios prosódicos não implicam o isolamento do componente morfológico - os domínios prosódicos são baseados indiretamente nas informações morfológicas. De acordo com a FLP do PB (Lee, 1995), o léxico do PB tem dois níveis ordenados - nível derivacional $(\alpha)$ e nível flexional $(\beta)$.

Pode-se postular, inicialmente, que a formação do diminutivo envolvendo o elemento -inho é um processo derivacional (-inhoé, portanto, um sufixo), ao passo que a formação do diminutivo envolvendo o elemento -zinho é um processo de composição ${ }^{6}$ (-zinho é, portanto, considerado como uma palavra fonológica). Em primeiro lugar, em relação ao processo que envolve o sufixo -inho, há alguns problemas:

i) os processos derivacionais se sujeitam à regra de neutralização vocálica, como se observou em (4), enquanto o processo de formação com sufixo -inho não se sujeita a tal regra, como em (5);

ii) há regras de alomorfia nos processos derivacionais, tais como a regra de assibilação e a regra de abrandamento de velar, exemplificado em (9):

(9) a. paciente + ia $\rightarrow$ paciên[s]ia presidente + ia $\rightarrow$ presidên[s]ia

b. fonólo[g]o + ia $\rightarrow$ fonolo[Z]ia elétri[k]o + idade $\rightarrow$ eletri[s]idade

Mas essas regras não se aplicam com o sufixo -inho, embora se satisfaça a descrição das regras no nível $\alpha$, como mostra (10):

(10) pacien $\left[\mathrm{t} /{ }^{*} \mathrm{~s}\right]$ inho, eletri[k/*s]inho 
iii) os processos de formação de nova palavra (derivação) mostram que a categoria e o gênero são determinados pelo elemento à direita - núcleo à direita - enquanto a palavra formada com o sufixo -inho não apresenta essa propriedade - núcleo à esquerda, como foi observado na seção anterior.

Esses fatos, portanto, mostram que o sufixo de formação produtiva -inho não pode ocorrer no nível $\alpha$, junto aos sufixos derivacionais. Em outras palavras o sufixo -inho não pode ser o sufixo derivacional.

Em segundo lugar, no processo de formação produtiva, o sufixo -zinho, assim como o composto, apresenta propriedades similares a propriedades próprias dos compostos pós-lexicais, que são: i) a existência de plural entre os constituintes; ii) a concordância entre os constituintes (ou seja, entre radical derivacional e sufixo). No entanto, apesar dessas semelhanças, há algumas diferenças claras entre o sufixo zinho e os compostos pós-lexicais:

1a) A formação produtiva apresenta o deslocamento de acento, como em (11a), mas isso não ocorre nos compostos pós-lexicais e nas frases sintáticas, como em $(11 \mathrm{~b}, \mathrm{c})$ :

(11) a. café zínho $\rightarrow$ càfezínho jacaré zínho $\rightarrow$ jacárezínho

b. cirurgião-médico amór-próprio, * àmor-próprio azúl-fíno, *àzul-fíno

c. um computadór lénto, *um computàdor lénto

$2^{a}$ ) Na formação de plural, a formação produtiva sofre a regra de simplificação para satisfazer o Princípio da Preservação de Estrutura - na medida em que o PB não permite as seqüências /S z/ e /S s/ na sua estrutura fonológica durante operações lexicais - o que se verifica em (12 a); o composto pós-lexical e a frase sintática, entretanto, não se sujeitam ao princípio de preservação de estrutura (cf. Lee, 1995, 1997b), como mostram os exemplos de (12 b, c): 
(12) a. cafes zinhos $\rightarrow$ cafezinhos

hoteis zinhos $\rightarrow$ hoteizinhos

b. médicos-[s]irurgiões

c. pa[s] sólida

Os exemplos de $(11,12)$ mostram que o sufixo -zinho não pode ser considerado como o composto pós-lexical, embora demonstre semelhanças. Em outras palavras, a sufixação -zinho não pode acontecer no nível pós-lexical, onde acontece o composto pós-lexical.

Os fatos mencionados acima evidenciam que os sufixos de formação produtiva do PB não pertencem ao nível $\alpha$, nem ao nível pós-lexical - a formação produtiva ocorre depois do nível $\alpha$ e antes do nível pós-lexical, de modo que, possivelmente, a formação produtiva ocorre no nível $\beta$. Essa hipótese mostra-se plausível diante de alguns fatos, conforme se pode ver, a seguir.

Para ocorrer no mesmo nível, os sufixos de formação produtiva têm de compartilhar semelhanças, o que não falta:

i) as propriedades morfológicas são iguais, embora a distribuição de sufixação seja determinada fonologicamente, como se observou na seção anterior;

ii) as vogais médias baixas do radical derivacional não se sujeitam à regra de neutralização vocálica, embora fiquem na posição átona, como em (5).

No nível $\beta$, ocorrem as flexões verbais regulares, que mostram propriedades semelhantes às do processo de formação produtiva considerando a noção de núcleo da palavra (cf. Di Sciullo \& Williams, 1987), o verbo possui o núcleo à esquerda; além disso, não há regra de neutralização vocálica na posição átona nesse nível. Assim sendo, pode-se postular que a formação produtiva ocorre no nível $\beta$, junto às flexões verbais regulares.

Mas, se isso é verdade, o acento da formação do não-verbo é problemático, na medida em que, por enquanto, se assume que a regra de acento do não-verbo se aplica no nível $\propto$. Uma solução para 
esse problema é assumir que a regra de acento se aplica aos sufixos de nível $\beta$, já que os sufixos também são entradas lexicais (cf. Lieber 1980), como representado em (13):

(13) Nivel $\propto$

/sapato/ /inho/ /cafE/ /zinho/

sapáto ínho café zínho acento

Nivel $\beta$

sapáto ínho cafézínho

sapatínho

$\overline{\text { sapatínho }}$

$\overline{\text { cáfEzínho }}$

Sufixação

Desacentuação

Deslocamento do acento

Entretanto, (13) prevê que o domínio prosódico do sufixo -zinho difere do domínio prosódico do sufixo -inho, uma vez que apenas o primeiro contém dois acentos na sua estrutura; isso significa, portanto, que os sufixos de formação produtiva do PB são subcategorizados morfológica e prosodicamente, como em (14):

(14) a. Domínio morfológico

$$
\text { -inho, -zinho: }\left\langle<>_{\mathrm{m} \beta}\right\rangle_{\mathrm{m} \beta}
$$

b. Domínio prosódico

$$
\begin{array}{ll}
\text {-inho: } & {\left[[]_{\mathrm{p} \beta}-\right]_{\mathrm{p} \beta}} \\
\text {-zinho: } & {[]_{\mathrm{p} \beta}[]_{\mathrm{p} \beta}}
\end{array}
$$

Além disso, a presença de um morfema plural entre o radical derivacional e o sufixo -zinho significa que a sufixação ocorre depois da formação do plural. Portanto, os domínios prosódicos e morfológicos da formação produtiva podem ser representados como em (15):

(15) Nivel $\alpha$

/sapato iño/ /kafe ziño/ /otel ziño/

$<$ sapato $><$ iño $>\quad$ kafe $><$ ziño $><$ otel $><$ ziño $>$

$\mathrm{MCF}$

[sapat]o [iñ]o

[kafe] [ziñ]o [otel] [ziñ]o

PCF

[sapát]o [íñ]o

[kafé] [zíñ]o [otél] [zíñ]o

Acento 
Nível $\beta$

$<$ sapato $><$ iño $>\quad<$ kafe $><$ ziño $><$ otel $><$ ziño $>$ MCF

[sapáto] [íño]

$<$ sapatiño>

[sapatíño]

[kafé] [zíño] [otél] [zíño] PCF

[sapatíño]
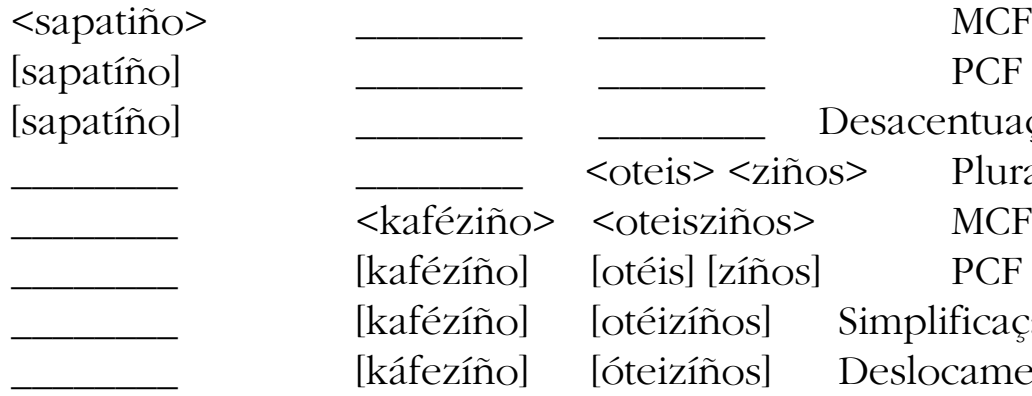

Desacentuação $<$ oteis $><$ ziños $>\quad$ Plural

$<$ kaféziño> $<$ oteisziños $>\quad$ MCF [kafézíño] [otéis] [zínos] PCF [kafézíño] [otéizíños] Simplificação [káfezíño] [óteizínos] Deslocamento de acento $\begin{array}{ccc}: & : & \\ \text { [sapatíño] } & & \\ \text { [kàfezíno] [òteizíños] }\end{array}$

\section{CONCLUSÃO}

Este trabalho mostrou que a formação de diminutivo mostra as caraterísticas diferentes da derivação, da flexão e do composto, ou seja, essa formação tem estatuto independente na gramática. Em relação ao seu domínio de aplicação, argumentou-se que essa formação de diminutivo acontece no nível $\beta$ - onde se aplica a formação flexional, (enquanto os processos derivacionais/compostos lexicais acontecem no nível $\alpha$ ), diferenciando-se do compostos póslexicais, que acontecem no nível pós-lexical. 


\section{NOTAS}

${ }^{1}$ No entanto cabe ressaltar que, na fala cotidiana, há variações, tais como: lampadazinha $\sim$ lampinha $\sim$ lampadinha, numerozinho $\sim$ numerinho, arvorezinha $\sim$ arvinha, chacarazinha $\sim$ chacrinha, facilzinho $\sim$ facinbo, papelzinbo papelinho, etc.

${ }^{2}$ Em alguns dialetos do $\mathrm{PB},[\mathrm{E}, \mathrm{O}]$ ocorre na posição pretônica, como d[O]larização, plOleira. Esses dialetos não apresentam contraste de [e, o] vs. [E, O] na posição pretônica, de modo que há 5 vogais [a, E, i, O, u] nessa posição pela neutralização vocálica (abaixamento vocálico).

${ }^{3}$ De acordo com Lee (1995), há dois tipos de acento secundário no PB - por exemplo, em pindamònhangába, o acento secundário é determinado pelo ritmo, enquanto, em guàrda-chúva, o acento secundário é determinado pela regra de acento lexical.

${ }^{4}$ A forma de plural contendo a flexão entre o radical e o sufixo é a forma adotada como padrão, mas, na fala cotidiana, exemplos como hotelzinhos e marzinhos são muto bem aceitos; parece, inclusive, que a tendência do PB atual é evitar (perder ??) a flexão entre o radical derivacional e sufixo -zinho.

${ }^{5}$ Hipótese de Referência Indireta (Indirect Reference Hypothesis) (Inkelas, 1993:77): "Phonological rules have access only to p-structure (i.e. not to mstructure or c-structure)".

${ }^{6}$ Este trabalho, seguindo Lee (1997a), assume que, no PB, há dois tipos de compostos: compostos lexicais e compostos pós-lexicais. Os compostos lexicais são formados no nível junto com os processos derivacionais e os compostos póslexicais são formados no componente pós-lexical. Os compostos lexicais mostram as mesmas caraterísticas dos processos derivacionais.

7 De acordo com Lee (1997b), as seqüências de /S s/ e /S z/, encontradas nos processos derivacionais e a pluralização da formação produtiva (-zinho), essas seqüências não são consoantes geminadas, uma vez que o vozeamento de /S/ não está especificado na representação subjacente. Mas observe-se que essas seqüências compartilham todos os traços, exceto o traço [sonoro]. 


\section{REFERÊNCIAS BIBLIOGRÁFICAS}

BRAKEL, A. Boundaries in a morphological grammar of Portuguese. Word 32, p.193-212, 1981.

CÂMARA Jr, M. Estrutura da Lingua Portuguesa. Petrópolis: Vozes, 1970.

DI SCIULlO, A. M. \& E. Williams. On the definition of word. MIT Press, 1987.

INKELAS, S. Prosodic costituency in the lexicon. Doctoral Dissertion. Stanford University. Stanford, 1989.

INKELAS, S. Deriving Cyclicity, in Hargus, S. \& E. M. Kaisse (eds.) The Studies in lexical phonology. San Diego: Academic Press, 1993.

LEE, S.-H. Fonologia lexical do Português. Cadernos de Estudos Lingüísticos 23, Campinas: Unicamp - IEL, 1992.

LEE, S.-H. Morfologia e fonologia lexical do Português do Brasil. Tese de Doutorado Unicamp - IEL Campinas, 1995.

LEE, S.-H. Sobre os compostos do PB, em DELTA 13-1, p.17-36, 1997a.

LEE, S.-H. Anti-geminação e silabificação no Português. Estudos Lingüisticos XXVI Anais de Seminários do GEL, p.581-587, 1997b.

LEITE, Y. Portuguese stress and related rules. Doctoral dissertation, University of Texas at Austin, 1974.

LIEBER, R. On the organization of the lexicon. Doctoral dissertation, MIT, 1980.

MORENO, C. Os diminutivos em -inho e -zinho, e a delimitação do vocábulo nominal em Português. Dissertação de Mestrado, IL/UFRGS, 1977.

MENUZZI, S. On the prosody of the diminutive alternation -inbo/-zinbo in Brazilian Portuguese. Ms. HIL/University of Leiden, 1993.

SIEGEL, D. Topics in english morphology. Doctoral dissertation. MIT, 1974.

WETZELS, W. Leo. Contrastive and allophonic properties of brazilian portuguese vowels. Paper presented at LSRL 18, University of Illinois at Urbana-Champaign. (In: D. Kibbee \& D. Wanner (eds.) New Analyses in Romance Linguistics, Amsterdam: J. Benjamins, 1988.)

WETZELS, W. Leo. Harmonização vocálica, truncamento, abaixamento e neutralização no sistema verbal do Português: uma análise auto-segmental. Cadernos de Estudos Linguísticos, UNICAMP, Campinas, 1991.

WETZELS, W. Leo. Mid vowel alternations in the brazilian portuguese verb. Phonology 12. p.281-304, 1995. 